\title{
Meta
}

Journal des traducteurs

Translators' Journal

\section{PIERRON, Agnès, avec la collaboration de Laurence LAPORTE et Didier LE BONHOMME (1991) : Dictionnaire de citations \& jugements, Coll. Les usuels du Robert, Paris, Dictionnaire Le Robert, XXII + 1313 p.}

\section{Jean-Yves Dugas}

Volume 37, numéro 3, septembre 1992

URI : https://id.erudit.org/iderudit/002671ar

DOI : https://doi.org/10.7202/002671ar

Aller au sommaire du numéro

Éditeur(s)

Les Presses de l'Université de Montréal

ISSN

0026-0452 (imprimé)

1492-1421 (numérique)

Découvrir la revue

Citer ce compte rendu

Dugas, J.-Y. (1992). Compte rendu de [PIERRON, Agnès, avec la collaboration de Laurence LAPORTE et Didier LE BONHOMME (1991) : Dictionnaire de citations \& jugements, Coll. Les usuels du Robert, Paris, Dictionnaire Le Robert, XXII + 1313 p.] Meta, 37(3), 550-552. https://doi.org/10.7202/002671ar d'utilisation que vous pouvez consulter en ligne. 
PIERRON, Agnès, avec la collaboration de Laurence LAPORTE et Didier LE BONHOMME (1991): Dictionnaire de citations \& jugements, Coll. Les usuels du Robert, Paris, Dictionnaire Le Robert, XXII + 1313 p.

Si l'on dispose de nombreux recueils de pensées, de florilèges poétiques, de morceaux choisis des grands auteurs, il n'en va pas ainsi pour le domaine encore peu prospecté de l'onomastique. En effet, les noms de personnes et les noms de lieux, bien qu'ayant suscité certaines études sur leur nature, leur rôle dans l'économie générale de la langue, leur origine, fort peu de travaux ont porté sur leur place, par ailleurs significative, dans l'ensemble de la pensée humaine. Scrutés comme objets de dénomination, d'identification, ils ont été généralement laissés pour compte comme vecteurs de la rêverie ou du savoir, comme interrogateurs de l'esprit, de l'âme, comme stimulateurs de la sensation ou de l'imagination, l'œuvre de Proust constituant cependant une exception à cet égard.

Le magnifique ouvrage d'Agnès Pierron et de ses collaborateurs vient combler une importante lacune sous cet angle. On y trouve plus de 9900 jugements, pensées, opinions, évaluations, réactions, impressions... choisis avec soin, qui prennent pour objet plusieurs centaines de personnes, de personnages, de lieux, d'événements, d'ouvrages, de monuments, d'activités, etc., ici rassemblés en une impressionnante fresque aux multiples chatoiements. L'inventaire nous conduit d'Abbeville à Stefan Zweig en passant par la Grande-Bretagne et Nicolas Poussin, par Québec et Marie-Claire Blais, par le Vatican et Confucius. Au gré de sa fantaisie, le lecteur est invité à des bonds prodigieux dans l'espace (de l'Amérique à l'Australie, de Malines à Tombouctou), dans le temps (de l'Antiquité à aujourd'hui), dans l'activité humaine (de la littérature à l'ingénierie, de la musique au journalisme, de la politique à la mythologie), qui permettent une vision kaléidoscopique de l'humanité en ses manifestations essentielles.

Ce foisonnement est toutefois offert de manière rationnelle en privilégiant la présentation des unités onomastiques objets de commentaires retenues suivant l'ordre alphabétique. Chacune des citations est référencée de telle sorte que la personne qui désirerait en connaître davantage sur tel lieu ou tel personnage pourra aisément retracer le passage cité. Les jugements ou les extraits sont ordonnancés chronologiquement et parfois 
accompagnés d'un renvoi à une autre rubrique: témoins Sallenches, Sévigné, Vatican. La liste complète des ouvrages dépouillés clôt le dictionnaire et est suivie d'un index des auteurs des citations avec référence au numéro de l'extrait concerné. Ainsi, malgré une masse documentaire considérable, la consultation de l'ouvrage demeure toujours aisée.

Rendre compte de manière complète de la richesse d'une si remarquable collection relève de la présomption et requiert une obstination sisyphienne que l'espace ici disponible nous épargnera de démontrer. Tout comme l'auteur, nous illustrerons l'intérêt de ce livre en exerçant arbitrairement un choix porté par un examen sélectif et forcément superficiel. Ce qui frappe d'emblée, c'est l'extraordinaire raccourci de la pensée qui cerne en quelques mots ou en plusieurs lignes un être, un lieu, un phénomène. Pour Paul Léautaud, le moraliste Vauvenargues n'est qu'un endormeur (p. 1212) alors qu'il faut à Darius Milhaud 25 lignes pour décrire un Erik Satie malade (p. 1065). Pour Victor Hugo, «la Belgique est le pays du monde où les maisons sont les plus propres et les femmes les plus sales» (p. 116), alors que James Joyce résume l'entreprise d'exploration colombienne ainsi: «Comme chacun sait, Christophe Colomb est devenu célèbre pour avoir été le dernier à découvrir l'Amérique.» (p. 289).

Ce recueil véhicule les plus subtiles pensées, les plus éclatantes descriptions, les plus délicates impressions, comme les observations tronquées, les jugements à l'emportepièce, les règlements de compte en règle. Se côtoient le meilleur et le pire, l'encensoir et le coup de trique, la verbosité et le sens de la formule qui, dans l'ensemble, se rééquilibrent, se nuancent, se replacent en perspective. Par exemple, le poète Paul Valéry fait l'objet du persiflage de Claudel («Extrême bassesse et grossièreté des pensées» p. 1202) et de l'admiration de Guéhenno qui estimait que «tout changeait, devenait intelligent quant il était là» (p. 1203). Il en va ainsi des lieux, Percy Bysshe Shelley soutenant que: «l'Enfer est une ville très semblable à Londres» (p. 704), pendant que Philippe Soupault s'émerveille: «Quelle ville et surtout quel port! Le Rhin m'avait émerveillé, Londres me donna le vertige» (p. 706).

Le Canada et le Québec occupent aussi leur place dans le panorama offert, notamment des lieux comme 1'Acadie, Banff, Calgary, Canada, le Cap-Breton, la baie d'Hudson, les Laurentides, Montréal, La Nouvelle-France, l'Île-du-Prince-Édouard, Québec, les Rocheuses, le Saguenay, le lac Saint-Jean, le Saint-Laurent, Terre-Neuve, Trois-Rivières, Vancouver, Winnipeg, ou des écrivains comme Hubert Aquin, Philippe Aubert de Gaspé, Marie-Claire Blais, Gérard Bessette, Robert Choquette, Réjean Ducharme, Louis Fréchette, Albert Laberge, Gratien Lapointe, Gaston Miron, Gabrielle Roy, ou un politicien comme Maurice Duplessis. On y découvrira des textes connus comme ceux du père Charlevoix qui retrace l'étymologie de Québec ou du mystérieux J.C.B. qui compare Québec et Montréal, d'autres plus rares de Jean Éthier-Blais sur monseigneur Camille Roy ou de Victor-Lévy Beaulieu sur Duplessis. Pour le lecteur québécois qui demeurerait sur sa faim, nous suggérons, en complément, les journaux de voyage ou les rapports de mission de l'époque de la Nouvelle-France au XIX ${ }^{\mathrm{e}}$ siècle, les œuvres de Gabriel Sagard, Sixte Le Tac, Pierre Boucher, les Relations des Jésuites, les Nouveaux voyages en Amérique septentrionale du baron de Lahontan (1703), le Journal de voyage de Pehr Kalm (1749), le Journal de Saint-Denys-Garneau, etc. A l'aide de ces textes, on pourrait constituer un véritable Pierron québécois, mais cela est une autre question.

La valeur et l'intérêt de ce dictionnaire résident surtout dans le fait de réunir en un même ouvrage de dimension raisonnable un panorama ondoyant et divers de l'intelligentsia et de l'espace universels. On y apprend maintes choses sur Shakespeare et Isabelle Adjani, sur les lieux Chioggia et Kimberley, à propos d'Eugénie Grandet et de l'océan Pacifique. Même s'il résulte de choix entachés de subjectivité, aspect qu'Agnès Pierron 
reconnaît volontiers d'entrée en jeu, ce livre permet des rencontres inédites, des découvertes passionnantes, des confrontations originales avec ses propres connaissances, ses jugements, voire ses préjugés personnels. Au gré d'un itinéraire adapté aux goûts et aux disponibilités de chacun, on peut élaborer sa mosaïque culturelle individuelle qui transcende le temps et l'espace.

À notre sens, on n'a jamais fait la part aussi belle à l'anthroponymie et à la toponymie, envisagées comme objets de discours, de descriptions, de traitements stylistiques. Désormais, le Pierron compte parmi les grands ouvrages de la lexicographie onomasticoculturelle, adapté qu'il demeure à une époque où le condensé, la synthèse et le résumé dominent sans négliger les vertus de l'humanisme, du savoir, aspects ici admirablement conjugués. 